[Highlight]

\title{
原子尺度原位揭示纳米粒子相变微观机制
}

\author{
赵进才
}

(中国科学院化学研究所, 北京 100190)

\section{In situ Atomically Revealing the Microscopic Mechanism of Phase Transition in Single Nanoparticles}

\author{
ZHAO Jin-Cai \\ (Institute of Chemistry, Chinese Academy of Sciences, Beijing 100190, P. R. China)
}

凝固和熔化是自然界普遍存在的现象, 广泛 涉及到工业生产及物理、化学、生命、材料和环 境等科学研究领域。但是, 由于缺乏直接实验依 据, 目前关于液态、固态之间相变的微观机制认 识尚不十分清楚。为此, 在原子尺度观察材料相 变过程中的成核、生长等微观动力学过程则是认 识相变微观机制的关键。由于相变过程的不连续 性、瞬时性且发生的空间范围往往在原子尺度等 特点, 实验上很难实现在操控材料相变的同时对 其微观动力学过程进行全面和详细记录。因此, 相变微观动力学研究至今仍是一项具有挑战性的 工作。为了克服这一困难, 在实验上, 目前普遍 利用胶体粒子(粒径一般在微米量级)模拟实际物质 中的原子来构建胶体体系作为研究对象 ${ }^{1}$, 在该体 系中相变速率较慢且基本粒子粒径较大, 容易观 察到体系的相变过程。但是, 这种模拟体系相变 与实际相变仍可能存在较大差别。在纳米粒子 中, 一个独立的成核、生长过程通常能导致整个 纳米粒子的相变。因此, 单个纳米粒子是用来揭 示物质相变微观机制较为理想的研究对象。

中国科学院新疆理化技术研究所王传义、李 英宣等与美国犹他大学藏泠教授等合作, 在前期 $\mathrm{Bi}$ 纳米粒子制备和生长机制研究基础上 ${ }^{2,3}$, 通过原 位高分辨透射电镜, 利用固态和液态 $\mathrm{Bi}$ 导热性的 差异, 巧妙地实现了 $\mathrm{Bi}$ 纳米粒子在 $\mathrm{SrBi}_{2} \mathrm{Ta}_{2} \mathrm{O}_{9}$ 基底 上温度的自我调控, 进而实现了固态和液态 $\mathrm{Bi}$ 的
可逆相变。在原子尺度上原位实时观察到了固态 和液态可逆相变在成核之前、成核、生长的整个 微观动力学过程。在固-液相变过程中发现了以缺 陷作为成核位点的异相成核生长机制。在实验上 验证了Samanta 等 ${ }^{4}$ 通过理论计算提出的固-液转变 微观过程。在液-固相变中观察到了通过一种有序 液体中间态的两步机制, 为经典的一步机制提供 了有效补充。在原位观察基础上, 研究者进一步 提出了一种 “作用-松弛” 的介尺度相变模型。该 研究为液固可逆相变提供了一个新的微观结构演 化的物理图像, 为今后在理论和实验上深入理解 相变这一重要的科学问题提供了借鉴。相关研究 成果发表在 Nature Communications 上 ${ }^{5}$ 。题目为 In situ study on atomic mechanism of melting and freezing of single bismuth nanoparticles 。

\section{References}

(1) Wang, Z.; Wang, F.; Peng, Y.; Zheng, Z.; Han, Y. Science 2012, 338, 87. doi: 10.1126/science. 1224763

(2) Li, Y.; Zang, L.; Li, Y.; Liu, Y.; Liu, C.; Zhang, Y.; He, H.; Wang, C. Chem. Mater. 2013, 25, 2045. doi: 10.1021/cm400065x

(3) Li, Y.; Bunes, B. R.; Zang, L.; Zhao, J.; Li, Y.; Zhu, Y.; Wang, C. ACS Nano 2016, 10, 2386. doi: 10.1021/acsnano.5b07197

(4) Samanta, A.; Tuckerman, M. E.; Yu, T. Q.; Weinan, E. Science 2014, 346, 729. doi: 10.1126/science. 1253810

(5) Li, Y.; Zang, L.; Jacobs, D. L.; Zhao, J.; Yue, X.; Wang, C. Nat. Commun. 2017, 8, 14462. doi: 10.1038/ncomms 14462 